três ciclos de hiperestimulação ovariana controlada com hormônio foliculo-estimulante recombinante. Os objetivos do estudo foram: 1) avaliar os efeitos da eletrocauterização laparoscópica ovariana bilateral sobre o volume ovariano e as dosagens séricas do hormônio folículo-estimulante, hormônio luteinizante, testosterona, androstenediona e insulina; 2) determinar a associação entre as variáveis hormônio luteinizante > 12 UI, índice de massa corpórea, quociente glicemia de jejum / insulinemia de jejum e teste de tolerância à glicose oral e a taxa de gestação no grupo de mulheres submetidas à eletrocauterização laparoscópica ovariana bilateral e 3) comparar as taxas de gestação dos dois grupos de tratamento. Houve diminuição estatisticamente significativa do volume ovariano bilateral após a eletrocauterização laparoscópi- ca ovariana bilateral. Observou-se queda estatisticamente significativa dos níveis séricos de testosterona e androstenediona e uma tendência à queda na média dos niveis séricos de hormônio luteinizante. Não houve influência do nivel sérico de hormônio luteinizante, do indice de massa corpórea, do quociente glicemia/ insulina de jejum e do teste de tolerância à glicose oral na taxa de gestação após a eletrocauterização ovariana laparoscópica. A taxa de gestação obtida com a eletrocauterização ovariana laparoscópica, doze meses após o procedimento, não diferiu da taxa de gestação obtida com três ciclos de indução ovulatória com hormônio folículo-estimulante recombinante.

Palavras-chave: Síndrome dos ovários policísticos. Eletrocauterização ovariana laparoscópica. Anovulação.

\title{
Estudo da Prevalência e dos Fatores Associados às Lesōes Intra-epiteliais Escamosas do Colo Uterino em Mulheres Infectadas pelo HIV
}

Autor: Ricardo José de Oliveira e Silva

Orientador: Prof. Dr. Aldo Franklin Ferreira Reis

Tese de Doutorado apresentada ao Programa de Pós-graduação em Clínica Médica, Setor de Pesquisa Clínica, da Universidade Federal do Rio de Janeiro - UFRJ, em 12 de maio de 2003.

Objetivos: Avaliar a prevalência de lesões escamosas intra-epiteliais cervicais em pacientes infectadas pelo HIV atendidas em rede pública na Cidade do Rio de Janeiro e estudar os fatores associados a essas lesões. Material e Método: 354 pacientes infectadas pelo HIV e atendidas em serviços públicos na Cidade do Rio de Janeiro foram submetidas a exame ginecológico, colheita de citologia e exame colposcópico do colo uterino e vulva. A associação do diagnóstico de lesão intra-epitelial do colo foi analisada de acordo com os resultados de variáveis clínicas (idade e presença de lesões vulvares), laboratoriais (contagem de CD4) e comportamentais (número de parceiros e hábito de fumar).

Resultados: A prevalência de lesões intra-epiteliais do colo uterino foi de $35,5 \%$. Na análise multivariada mostraram-se significativas: contagem de CD4 abaixo de 350 céls $/ \mathrm{mm}^{3} \mathrm{OR}=2,31$ [IC 95\% 1,43 - 3,75], hábito de fumar OR = 1,83 [IC 95\% 1,13 - 2,97], a lesão intra- epitelial vulvar OR = 2,28 [IC 95\% 1,41 - 3,70] e a idade abaixo de 33 anos OR = 1,74 [IC 95\% 1,07 - 2,82]. Na análise do subgrupo das lesões de alto grau mostraram-se significativas: contagem de CD4 abaixo de 350 céls $/ \mathrm{mm}^{3}$ OR = 2,62 [IC 95\% 1,13 - 6,10], hábito de fumar OR = 2,72 [IC 95\% 1,20 - 6,21] e idade abaixo de 33 anos OR = 2,56 [IC 95\% 1,10 - 5,96].

Conclusões: É alta a prevalência de lesões intraepiteliais do colo uterino em pacientes infectadas pelo HIV. A imunodeficiência, o hábito de fumar, a presença de lesões intra-epiteliais na vulva e a idade abaixo de 33 anos estão associadas à presença de lesões intraepiteliais do colo uterino. Excetuando-se as lesões vulvares, a mesma associação foi encontrada para as lesões intra-epiteliais de alto grau.

Palavras-chave: AIDS. Colo: lesões pré-neoplásicas. Tabagismo. Epidemiologia. 